\title{
ARTIGOS
}

ESPAÇO, ECONOMIA E POPULAÇÃO

\section{ESTRUTURA ESPACIAL E PROVISÃO DE ATENÇÃO PRIMÁRIA À SAÚDE NOS MUNICÍPIOS BRASILEIROS}

\author{
Pedro Amaral* \\ Lucas Resende de Carvalho* \\ Luciana Luz* \\ Allan Claudius Queiroz Barbosa** \\ *Universidade Federal de Minas Gerais, Centro de Desenvolvimento e Planejamento Regional, Belo Horizonte, \\ MG, Brasil \\ **Universidade Federal de Minas Gerais, Centro de Pós-graduação e Pesquisas em Administração, Belo Horizonte, \\ MG, Brasil
}

\section{Resumo}

Este artigo discute o papel da distância geográfica na provisão de serviços de Atenção Primária à Saúde (APS) no Brasil, considerando a oferta de unidades básicas de saúde e de equipes de atendimento nessa área em municípios brasileiros. Para identificar a cobertura de serviços de APS, foram utilizados métodos de análise exploratória de dados espaciais, avaliação de equilíbrio entre oferta e demanda potencial (intermunicipal) e modelos de programação linear, com vistas a identificar setores censitários que concentram população coberta e descoberta (intramunicipal). Segundo os resultados obtidos, 6\% dos municipios brasileiros apresentam menos de 50\% da população coberta, enquanto algumas cidades apresentam mais equipes do que seria potencialmente necessário. Na análise intramunicipal, os resultados de estudo em capital brasileira - Belo Horizonte (MG) - apontam localidades onde as equipes de saúde podem não estar distribuídas de maneira equitativa. O que se observa, de forma geral, é que, mesmo com o recente aprimoramento da distribuição regional de médicos, ainda é possivvel verificar a ausência de provimento de APS em algumas localidades. A adoção de ações governamentais de provimento pode contribuir para a redução dessas disparidades regionais.

Palavras-chave

Cobertura de Serviços de Saúde; Atenção Primária à Saúde; Distribuição Regional; Equidade em Saúde. 


\author{
ARTICLES \\ SPACE, ECONOMY AND POPULATION
}

\title{
SPATIAL STRUCTURE OF PRIMARY HEALTH CARE PROVISION IN BRAZILIAN MUNICIPALITIES
}

\author{
Pedro Amaral ${ }^{*}$ \\ Lucas Resende de Carvalho* \\ Luciana Luz* \\ Allan Claudius Queiroz Barbosa** \\ *Universidade Federal de Minas Gerais, Centro de Desenvolvimento e Planejamento Regional, Belo Horizonte, \\ MG, Brasil \\ ** Universidade Federal de Minas Gerais, Centro de Pós-graduação e Pesquisas em Administração, Belo Horizonte, \\ MG, Brasil
}

\begin{abstract}
This article highlights the role of geographical distance in the provision of Primary Health Care (PHC) services in Brazil, considering the provision of PHC units and health care professionals in Brazilian municipalities. To identify the coverage of PHC services in Brazilian municipalities, methods of exploratory spatial data analysis, assessment of the balance between potential supply and demand, and linear programming models were used in order to identify census sectors that concentrate covered and uncovered population. The results show that $6 \%$ of Brazilian municipalities have less than $50 \%$ of the population covered, while some municipalities have more teams than would potentially be necessary. In the intra-municipal analysis, the results of a study in a Brazilian capital - Belo Horizonte $(M G)$ - point out locations where health teams may not be distributed in an equitable manner. Results show that, in general, that even in face of the recent improvement in regional distribution of doctors it is still possible to identify lack of provision of PHC in some locations. The adoption of government provision actions can contribute to the reduction of these regional disparities.
\end{abstract}

Keywords

Health Service Coverage; Primary Health Care; Regional Distribution; Equity in Health. 


\title{
ESTRUTURA ESPACIAL E PROVISÃO DE ATENÇÃO PRIMÁRIA À SAÚDE NOS MUNICÍPIOS BRASILEIROS'
}

\author{
Pedro Amaral \\ Lucas Resende de Carvalho \\ Luciana Luz \\ Allan Claudius Queiroz Barbosa
}

Introdução

Este artigo discute o papel, nos âmbitos inter e intramunicipal, da distância geográfica na provisão espacial de serviços de Atenção Primária à Saúde (APS) no Brasil, considerando a oferta de Unidades Básicas de Saúde (UBS) e de equipes de saúde em municípios brasileiros. Mais especificamente, busca-se identificar municípios e setores censitários em que a quantidade de equipes de saúde pode não ser suficiente para oferecer cobertura para a população, levando ainda em conta a distância entre população e serviços de saúde. Apesar dos avanços com vistas a aprimorar a distribuição regional de médicos e atender à necessidade de fixação dos profissionais (CARVALHO; MARQUES; SILVA, 2016), persistem cenários de ausência de provimento de serviços, áreas sem atendimento médico e parte da população fora da rede de atenção. Trata-se de um problema não aleatório espacialmente e, em geral, correlacionado ao nível de privação de outras variáveis socioeconômicas que indicam necessidade de cobertura.

Com efeito, ao identificar localidades onde existem "vazios” na rede de provisão, quantificando pessoas fora de áreas de cobertura potencial, cumpre-se o preceito constitucional de que é responsabilidade do Sistema Único de Saúde (SUS) garantir a todos os cidadãos brasileiros acesso aos serviços de saúde, com cobertura de suas necessidades de atenção e tratamento igualitário a pessoas com necessidades iguais.

1. Os autores agradecem à Fapemig pelo apoio recebido por meio do projeto APQ-04005-17. 
O cumprimento desse preceito torna-se ainda mais fundamental num cenário de agravamento epidemiológico, como é o contexto da pandemia da Covid-19, causada pelo novo coronavírus, SARS-CoV-2. Conforme estabelecido pelo Protocolo de Manejo Clínico da Covid-19, a Atenção Primária à Saúde/Estratégia Saúde da Família (APS/ESF) “deve assumir papel resolutivo frente aos casos leves e de identificação precoce e encaminhamento rápido e correto dos casos graves, mantendo a coordenação do cuidado destes últimos” (BRASIL, 2020, p. 6). A identificação e a análise de síndromes gripais pela APS são essenciais para que sejam realizados o diagnóstico correto e o encaminhando dos casos mais graves para os níveis de complexidade e regionalização adequados do sistema, com a manutenção dos casos menos graves na esfera da APS, sob acompanhamento dos profissionais ao longo do curso da doença (BRASIL, 2020; SERVO; ANDRADE; AMARAL, 2019). Tal estratégia só consegue atingir níveis satisfatórios de eficiência se toda a população tiver acesso aos serviços de APS/ESF.

O princípio é o acesso balanceado das pessoas aos serviços de saúde para recebimento de diagnóstico ou tratamento. Nesse contexto, acesso balanceado implica a possibilidade de acesso igualitário a pessoas com necessidades iguais. Portanto, o conceito de acessibilidade abrange mais que a simples existência ou a disponibilidade de recursos em dado período do tempo (ADAY; ANDERSEN, 1974); está também ligado à capacidade dos indivíduos de se apropriarem dos serviços oferecidos.

Sob a ótica da acessibilidade geográfica, a provisão espacial (ou configuração geográfica da rede de oferta) de serviços de saúde exerce papel fundamental, assim como o sistema de transporte. Conforme definido por Whitehead (1992), equidade em cuidado de saúde baseia-se no princípio de tornar acessíveis a toda a população serviços de saúde de qualidade. Isso significa que é necessário que o paciente seja capaz de alcançar o centro de oferta do serviço existente, ou ser por este alcançado, em tempo e custos razoáveis (HAMER, 2004). Portanto, há que se considerar uma rede de provisão de serviços de saúde geograficamente balanceada.

A configuração de uma rede de oferta de serviços de saúde requer uma avaliação da fricção espacial no acesso a esses serviços, em conjunto com uma análise da distribuição espacial dos centros de saúde, recursos e profissionais vis-à-vis à distribuição da população (JOSEPH; BANTOCK, 1982; KHAN, 2002). A oferta de tais serviços se beneficia da concentração espacial, em razão dos retornos crescentes de escala associados à provisão deles (POL; THOMAS, 2000; IUNES, 2002). Essa característica, aliada ao fato de profissionais de saúde geralmente preferirem habitar centros urbanos e áreas mais desenvolvidas (KUHN; OCHSEN, 2009; PÓVOA, ANDRADE; MORO, 2004), resulta na tendência de concentração de serviços de saúde em torno 
de aglomerados urbanos. Como consequência, sua presença é escassa em áreas relativamente menos desenvolvidas e rurais (AMARAL et al., 2017; ROCHA et al., 2017).

Uma vez que a condição de saúde é associada ao status socioeconômico (NORONHA, 2001) e a estrutura espacial da oferta de serviços de saúde impacta suas taxas de utilização (HIGGS, 2009), essa concentração é perversa no sentido de que a provisão de serviços de saúde é escassa em locais onde seria mais necessária: a chamada "inverse care law" (HART, 1971).

\section{Distribuição espacial dos serviços de saúde}

\subsection{O Sistema Único de Saúde}

O sistema de saúde brasileiro é misto, composto de dois subsistemas, o público, representado pelo SUS, e o privado. Antes da criação do SUS, a maior parte dos atendimentos públicos de saúde era realizada pelo Instituto Nacional de Assistência Médica da Previdência Social (Inamps). Por sua vez, o atendimento médico por ele prestado era restrito a grupos populacionais específicos que contribuíam com a previdência social. Com a implementação do SUS em 1988, a Constituição Federal brasileira passou a garantir o acesso gratuito, universal e integral aos serviços de saúde.

A criação do SUS foi o reconhecimento, pela população brasileira, de que o acesso equitativo e igualitário à saúde é um direito fundamental. Ainda é facultativo que a população faça uso do sistema de saúde privado, em que o acesso é realizado pelo desembolso direto (out-of-pocket payment) ou por meio da intermediação financeira de planos e seguros de saúde. Assim, a dualidade do sistema de saúde brasileiro é evidenciada pela Constituição de 1988, que, embora garanta um sistema de saúde público universal e gratuito, permite ao cidadão escolher utilizar o sistema privado. Em média, cerca de $75 \%$ de toda a população recebe assistência apenas na rede pública, enquanto os $25 \%$ restantes contam com alguma modalidade de seguro privado.

A expansão da atenção primária de qualidade no país é um passo fundamental para alcançar a Cobertura Universal de Saúde preconizada como um dos Objetivos de Desenvolvimento Sustentável da Organização das Nações Unidas (WHO, 2015). A principal política adotada, no Brasil, para a ampliação da atenção básica é o ESF, que tem como enfoque o cuidado preventivo e a promoção à saúde em detrimento do cuidado da agudização, com foco hospitalocêntrico. Além disso, as equipes de atenção básica ou as equipes de saúde da família atuam como a principal porta de entrada para o sistema público de saúde. 
Segundo Andrade et al. (2018b), esse desenho da atenção básica garante que todos os indivíduos sejam cadastrados e acompanhados dentro do sistema ao longo do tempo. As equipes provêm uma atenção básica abrangente que inclui o acompanhamento de doenças crônicas, a triagem para vacinação, o acompanhamento do pré-natal, o agendamento de horários nas UBS e também a coleta de informações socioeconômicas.

O dimensionamento da oferta de algum serviço de saúde pode ser definido em função do tamanho populacional ou da distância percorrida para o atendimento, com um número fixo de provedores para certo porte populacional ou uma distância máxima que o indivíduo deve cumprir para ser atendido. A oferta dos serviços de atenção básica não demanda recursos físicos de grande complexidade e, em geral, é mais intensiva em recursos humanos. Assim, a regulação do dimensionamento da sua oferta se baseia em um equilíbrio entre um nível mínimo de oferta de equipes dado o tamanho populacional.

No nível municipal, Andrade et al. (2018a) mostraram que, entre 1998 e 2012, o aumento da adoção e da cobertura, especificamente do ESF, não foi homogêneo entre os municípios. A implementação do programa foi positivamente associada ao baixo porte populacional, à baixa densidade populacional, à baixa cobertura de seguro saúde privado e ao baixo nível de desenvolvimento econômico. Além disso, o alinhamento do partido político do prefeito e do governador e a disponibilidade de recursos de saúde afetaram a adoção e a ampliação dessa oferta. Assim, justifica-se a focalização da atenção básica, conforme mostrado por Azevedo (2014), pois as condições precárias de moradia e a menor escolaridade e renda aumentam os riscos de problemas de saúde, impactando também a capacidade laboral daquela população e tornando-a ainda mais vulnerável a uma situação de pobreza.

Pereira et al. (2020b) mostram que, embora haja significativas diferenças de acessibilidade a oportunidades de emprego, serviços de saúde e educação entre os diferentes municípios brasileiros, as maiores desigualdades foram verificadas no espaço intraurbano, também por níveis de renda e cor/raça. Segundo os autores, a concentração das atividades em áreas urbanas centrais, combinadas com o espraiamento da população das maiores cidades brasileiras, favorece essa desigualdade.

Andrade et al. (2018b) concluem que a experiência brasileira de expansão da atenção primária à saúde oferece três lições quanto aos fatores que influenciam sua difusão. A primeira diz respeito ao fato de o mecanismo do financiamento ser fundamental para a implementação do programa a fim de apoiar a oferta de médicos de atenção primária em áreas de baixa densidade. Em segundo lugar, em áreas mais desenvolvidas e maiores, o principal desafio é a falta de incentivos para buscar cobertura universal, especialmente em face da maior disponibilidade de 
sistema de saúde privado. A terceira lição elencada pelos autores é que o tamanho da população constitui um elemento crucial para garantir a sustentabilidade da cobertura ao longo do tempo.

\subsection{Aspectos conceituais}

A cidade é definida por Ribeiro e Silva (2016) como um mosaico de espaços urbanos ricos e pobres, muito segregados, que produzem uma cidade heterogênea. Nela, a dinâmica espacial e populacional resulta em uma área nobre, que concentra serviços e infraestrutura, com áreas de população de baixa renda, carentes de moradia, emprego, infraestrutura básica e serviços de saúde.

A prestação de cuidados de saúde é composta de um espectro de serviços que variam em sua complexidade do básico ao muito complicado. Conforme proposto por Lösch (1954) para a economia geral, a provisão de serviços de saúde é organizada em torno de três polos: i) atividade humana, ii) processo de produção e iii) escolha de local. Os serviços básicos, como atenção primária à saúde, tendem a ser usados com mais frequência e têm custos mais baixos, enquanto serviços mais complexos em geral são mais sujeitos a economias de escala, pois envolvem maior tecnologia e menor densidade espacial de demanda (POL; THOMAS, 2000; IUNES, 2002). Por esse motivo, a distribuição dos serviços de saúde é espacialmente diferenciada e hierarquizada, de modo que o contexto urbano pode contribuir para o bem-estar populacional.

Dada a existência dessa diferenciação e dessa complexidade na oferta de serviços de saúde, é necessária a busca de elementos teóricos para interpretar a questão. A Teoria do Lugar Central (TLC), desenvolvida por Christäller (1966) e Lösch (1954), baseia-se no princípio da centralidade e leva em consideração o espaço a ser organizado em torno de um núcleo urbano principal, chamado de lugar central. A região complementar, ou entorno, apresenta uma relação de codependência com o núcleo principal, já que esse é o locus de oferta de bens e serviços de natureza urbana.

o principal papel de um núcleo urbano é ser um centro de serviços para o seu interior imediato, fornecendo bens e serviços essenciais. Assim, as cidades são organizadas em redes de tamanhos diferentes que se sobrepõem (LÖSCH, 1954). A TLC apresenta dois conceitos-chave: i) limite crítico, definido como o nível mínimo de demanda necessário para permitir que o fornecedor de um bem ou serviço obtenha lucros normais; e ii) alcance, definido como a distância máxima que o consumidor está disposto a percorrer para ter acesso a determinado bem ou serviço, e que varia conforme a complexidade do serviço.

Portanto, o limite crítico pode ser representado como o menor círculo concêntrico que justifica o fornecimento de um bem ou serviço, e o alcance pode ser 
descrito como o maior círculo concêntrico que forma a região complementar do lugar central e define sua área de influência. Os limites de tais áreas são dados pela existência de outra área de influência de outro centro de hierarquia semelhante ou superior. O tamanho desse círculo exterior varia de acordo com os diferentes bens e serviços fornecidos, e a demanda em seu interior varia inversamente em relação à distância até o núcleo urbano.

O modelo pretende demonstrar que o tamanho das áreas de influência de cada lugar central depende diretamente do tamanho e da hierarquia dos centros, em que a periferia dos centros menores é incluída nas regiões complementares das maiores. Quanto maior a centralidade de um lugar central, maior seu alcance - ou seja, quanto maior a complexidade dos serviços prestados, maior a área influenciada por esse centro.

No contexto de serviços públicos necessários, como cuidados de saúde, o equilíbrio entre os conceitos de limite crítico e alcance é complexo. Se uma região não apresentar certa quantidade mínima de população, o chamado limite crítico, a provisão de serviços de saúde de alta complexidade seria economicamente ineficiente. Como consequência, os habitantes de regiões menos povoadas não teriam acesso a uma variedade de serviços ou teriam que percorrer longas distâncias para receber assistência médica. Até que ponto uma distância é socialmente aceitável para um paciente viajar a fim de receber cuidados de saúde? Ou quando um limite crítico de eficiência econômica se torna socialmente injusto? Essas perguntas não têm resposta fácil ou clara, principalmente em face da universalidade constitucional do tipo de serviço aqui estudado. Guimarães (2001) aponta, além disso, estreita relação entre planejamento urbano e a saúde pública, em que o espaço social é influenciado pelas estatísticas de saúde, as quais se tornaram, segundo o autor, um poderoso instrumento de poder político.

Nesse contexto, a distribuição espacial da oferta de serviços de saúde é de suma importância porque impacta, diretamente, a distância coberta pelo paciente para efetivar seu acesso. Stock (1983), Dranove, William e Wu (1993), Goodman et al. (1997), Oliveira, Travassos e Carvalho (2004) e Amaral et al. (2017), dentre outros, destacam a importância da distância, e, por consequência, do tempo de deslocamento, na utilização de serviços de saúde. Logo, a distância percorrida e o tempo gasto podem se tornar uma barreira de acesso significativa, capaz de causar impacto na qualidade de vida da população e até de pôr em risco a vida de um paciente em situação mais complexa. Além disso, conforme mostrado por Azevedo (2014), a análise da estrutura dos serviços de saúde tem grande relevância na mensuração da capacidade de os indivíduos enfrentarem o risco de adoecer, pois as chances de sobrevivência, com algum grau de qualidade de vida, são afetadas, entre diversos fatores, pelo seu local de residência. 
Porém, como ressaltado por Amaral (2013), a disponibilidade do equipamento ou leito, por si só, não é suficiente para garantir o acesso da população. Segundo o autor, embora existam localidades com elevada oferta, o acesso real pode ser cerceado pela limitação de conhecimento de sua disponibilidade, pelo tempo de espera elevado, por barreiras culturais e por questões ligadas ao transporte ou à falta de pessoal técnico. Portanto, a disponibilidade de um equipamento não garante o acesso da população, ainda que, para que haja acesso, seja indispensável a existência da oferta.

Uma vez que a preocupação é com a distribuição geográfica da prestação atual de serviços de APS, a capacidade de cobertura por equipes de saúde é numericamente reduzida, de modo que se espera que sua oferta seja desconcentrada. Todavia, em escala intermunicipal ou intramunicipal, persistem no país disparidades espaciais na provisão de serviços de APS.

Em anos recentes, o Governo Federal adotou diferentes estratégias de provisão emergencial, tais como o Programa Mais Médicos, que contribuiu para o espraiamento de profissionais de saúde no território nacional, alcançando lugares que careciam de provisão (BARBOSA et al., 2018). Em 2019, havia no Brasil uma cobertura potencial de cerca de 150 milhões de pessoas (SAPS, 2020).

À luz do que foi exposto conceitualmente, este estudo utilizou como base geográfica municípios brasileiros com vistas a identificar quais apresentam maior ou menor nível de provisão de equipes de saúde. Posteriormente, foi feita uma análise na escala intramunicipal, em relação à qual a expectativa é de que os serviços sejam concentrados no espaço, gerando economias e ganhos de escala.

\section{Método}

Dada a concentração espacial da provisão, procurou-se identificar os desequilíbrios espaciais por ela apresentados, isto é, em quais regiões o nível de atendimento é maior do ponto de vista de oferta espacial e quais regiões se encontram em vazios da rede de oferta ou estão distantes dos pontos de provisão, tanto no nível inter como no intramunicipal.

Para a análise em nível municipal, foram consideradas 45.075 equipes de saúde conforme informação disponibilizada pelo Departamento de Atenção Básica (DAB), relativa a outubro de 2018. Os dados de população empregados para as análises nesse âmbito integram a estimativa para 2018 divulgada pelo Instituto Brasileiro de Geografia e Estatística (IBGE).

Quanto à análise intramunicipal, considerou-se, em outubro de 2018, o número de 42.670 equipes de saúde distribuídas em 30.360 UBS. Esse número é relativo às unidades de saúde com geolocalização informada corretamente pelo DATASUS (Departamento de Informática do SUS) ou cuja localização foi possível 
corrigir (ROCHA et. al., 2018). A análise intramunicipal foi baseada nos setores censitários do Censo Demográfico 2010, ${ }^{2}$ de maneira que se utiliza como dado a população daquele ano. No tocante à capacidade de atendimento das equipes de saúde, a Portaria no 2.436, de 21 de setembro de 2017, define que a população adscrita por equipe de atenção básica e de saúde da família deve ser de 2 mil a 3,5 mil pessoas, localizada dentro do seu território, garantindo, assim, os princípios e as diretrizes da Atenção Básica. Por esse motivo, neste estudo trabalha-se com uma capacidade de cobertura para 3.450 pessoas por equipe de saúde.

Assumindo o tamanho da população ( $p$ ) como uma medida da demanda e a quantidade de equipes de saúde (s) como uma medida de oferta, é possível estimar o excesso de demanda ou oferta $(E)$ de cada município $i$ :

$$
E_{i}=3.450 * s_{i}-p_{i}
$$

Para verificar se o padrão municipal encontrado é de fato significativo, será utilizado o indicador I de Moran Local para o percentual da população coberta. Esse indicador permite identificar a presença de agrupamentos espaciais de municípios com valores semelhantes ou diferentes para a variável em questão (ANSELIN, 1995). O indicador pode ser definido por:

$$
I=\frac{\left\lfloor\sum_{i} \sum_{j} \frac{w_{i j} z_{i} z_{j}}{S_{0}}\right\rfloor}{\left[\frac{\sum_{i} z_{i}^{2}}{N}\right]}
$$

em que z representa o percentual de população coberta nas localidades $i$ ou $j, \mathrm{~N}$ indica o número de observações e $\mathrm{w}_{\mathrm{ij}}$, se $i$ e $j$ são regiões vizinhas, critério aqui definido como regiões contíguas, isto é, vizinhança do tipo Rainha de primeira ordem.

O I de Moran pode ser interpretado como um coeficiente de correlação, cujo espaço amostral depende das características do critério de vizinhança. Quando, segundo o indicador, um município pertence a um grupo Alto-Alto, tem-se que essa localidade possui alto índice de cobertura por equipes de saúde e que seus vizi-

2. A utilização de dados do Censo Demográfico 2010 em escala intramunicipal é uma limitação relevante para este trabalho em virtude de sua defasagem. A dinâmica intramunicipal da população e da renda pode ter causado variações significativas nos dados. Todavia, optou-se por utilizá-los com o propósito de manter uma compatibilidade entre dados de população e renda e de não introduzir erros de estimativa de modelos de projeção na modelagem aqui adotada. 
nhos são municípios que, em média, também apresentam alto índice de cobertura. De maneira análoga, isso se aplica igualmente a valores Baixo-Baixo. Nos casos em que um município apresenta valores Alto-Baixo, o nível de cobertura no município é significativamente maior que a média de seus vizinhos. Da mesma forma, os valores Baixo-Alto apresentam localidades de baixos níveis de cobertura com vizinhos com alta cobertura.

No nível intramunicipal, a oferta de serviços é espacialmente concentrada nas UBS, que atendem não apenas a população dos setores em que se encontram, mas também a do seu entorno, até que toda a população seja coberta pela área de atendimento de uma UBS ou que se esgote a capacidade de atendimento. A área de cobertura dos serviços de APS pode ser estimada com o emprego de modelos de otimização linear. Esses métodos têm sido amplamente utilizados para resolver problemas de alocação em serviços de saúde (RAHMAN; SMITH, 2000), por exemplo. Com base na solução de um modelo de programação linear, é possível identificar os setores censitários que concentram população coberta e descoberta.

O problema de otimização deste estudo é composto de uma única variável de decisão: a quantidade de pessoas de cada setor censitário cobertas por UBS, de acordo com o número de equipes localizadas nessas unidades de atendimento. Visto que a variável de decisão compreende a quantidade de pessoas, esta não pode ser fracionada, ou seja, todas devem ser representadas por números inteiros. Como resultado, a otimização é do tipo Integer Linear Programming (ILP). O software Gurobi foi usado para resolver o seguinte problema de otimização:

Mínimo de

$$
\sum_{i} \sum_{j} d_{i j} h_{i j}
$$

Sujeito a

$$
\begin{aligned}
& \sum_{j} h_{i j}=p_{i}, \forall i, \\
& \sum_{i} h_{i j} \leq 3.450 * s_{j}, \forall j, \\
& h_{i j} \geq 0, \forall i, j .
\end{aligned}
$$

em que $i$ indica o setor censitário de residência da população, $j$ corresponde aos centros de provisão, ou seja, as UBS, $\mathrm{d}_{i j}$ é a distância em quilômetros entre o centroide do setor $i$ e a UBS $j$, $\mathrm{p}_{i}$ é o tamanho da população em $i$, e a variável de decisão $h_{i j}$ é a população do setor censitário $i$, coberta por equipe de saúde localizada na UBS $j$. 
Nessa solução, o objetivo da Equação 2 é minimizar a distância total ponderada entre as UBS $j$ e a população em $i$. As restrições da Equação 3 garantem que toda a população seja atendida por um centro de provisão, caso haja equipes suficientes para isso. As restrições da Equação 4 especificam o limite de capacidade de cobertura de cada UBS, ao passo que as restrições da Equação 5 impõem valores não negativos à variável de decisão.

Um grupo de restrições no modelo merece atenção especial. Trata-se da Equação 3, cujas restrições garantem que toda a população seja atendida por alguma UBS. No entanto, alguns municípios não dispõem de equipes suficientes para atender toda a população, o que significa que há excesso de demanda. Nesse caso, para que as restrições da Equação 3 sejam atendidas, a solução é inexistente.

Para resolver esse problema, modificam-se as restrições das Equações 3 e 4 . Em vez de exigir que toda a população seja atendida, assegura-se que toda a capacidade de cobertura seja esgotada. Algebricamente, as Equações 3 e 4 são substituídas, respetivamente, por $\sum_{j} h_{i j} \leq p_{i} \forall i$ e $\sum_{i} h_{i j}=3.450 * s_{j}, \forall j$.

Assim, a distância ponderada resultante nesses casos refere-se apenas à parcela da população que é servida. A população descoberta não afeta o resultado. Em face da minimização da distância ponderada total, quando não há equipamentos suficientes para atender a toda a população, a parcela da população que está mais distante dos centros de provisão não é alocada a nenhum outro centro e fica assinalada como população descoberta.

\section{Resultados}

3.1. Análise municipal

Na Figura 1, é possível visualizar os percentuais de cobertura para os 5.570 municípios brasileiros, demonstrando uma distribuição espacial da cobertura da população por equipes de saúde muito desigual no Brasil. É evidente não só a desigualdade interestadual, como também a intraestadual, haja vista que em diversos estados se verificam, ao mesmo tempo, municípios com excesso de demanda e outros com excesso de oferta.

Do total dos 5.570 municípios, 336 (6\%) têm menos de 50\% da população coberta. Desses, 57, localizados principalmente nos estados de São Paulo e Rio Grande do Sul, não contam com nenhuma equipe de saúde e apresentam $0 \%$ de cobertura. Do outro lado desse espectro, 3.879 municípios (69,6\%) dispõem de mais equipes do que seria necessário, se considerarmos o valor máximo de demanda potencial estimada de 3.450 pessoas por equipe. Desse total, 586 (10,5\%) apresentam quantidade de equipes mais que suficiente para atender à demanda potencial estimada, ainda que se contabilizasse o mínimo de pessoas por equipes preconizado pela 
Portaria $n^{0}$ 2.436/2017, ou seja, 2 mil, evidenciando alta concentração de equipes nessas localidades.

Cabe ressaltar que, ao se falar em quantidade de equipes em relação ao potencialmente necessário, não estão sendo levados em consideração aspectos como especificidades epidemiológicas, distância a ser percorrida, barreiras geográficas ou de acessibilidade, ou nenhum outro fator que pode ser crucial para reduzir a capacidade máxima de cobertura potencial de dada equipe de saúde. Todos esses fatores podem fazer com que a cobertura aqui considerada esteja sobre-estimada. Portanto, o foco deste trabalho é sobretudo a identificação de regiões que, mesmo sob o risco de sobre-estimação da cobertura, apresentam vazios assistenciais.

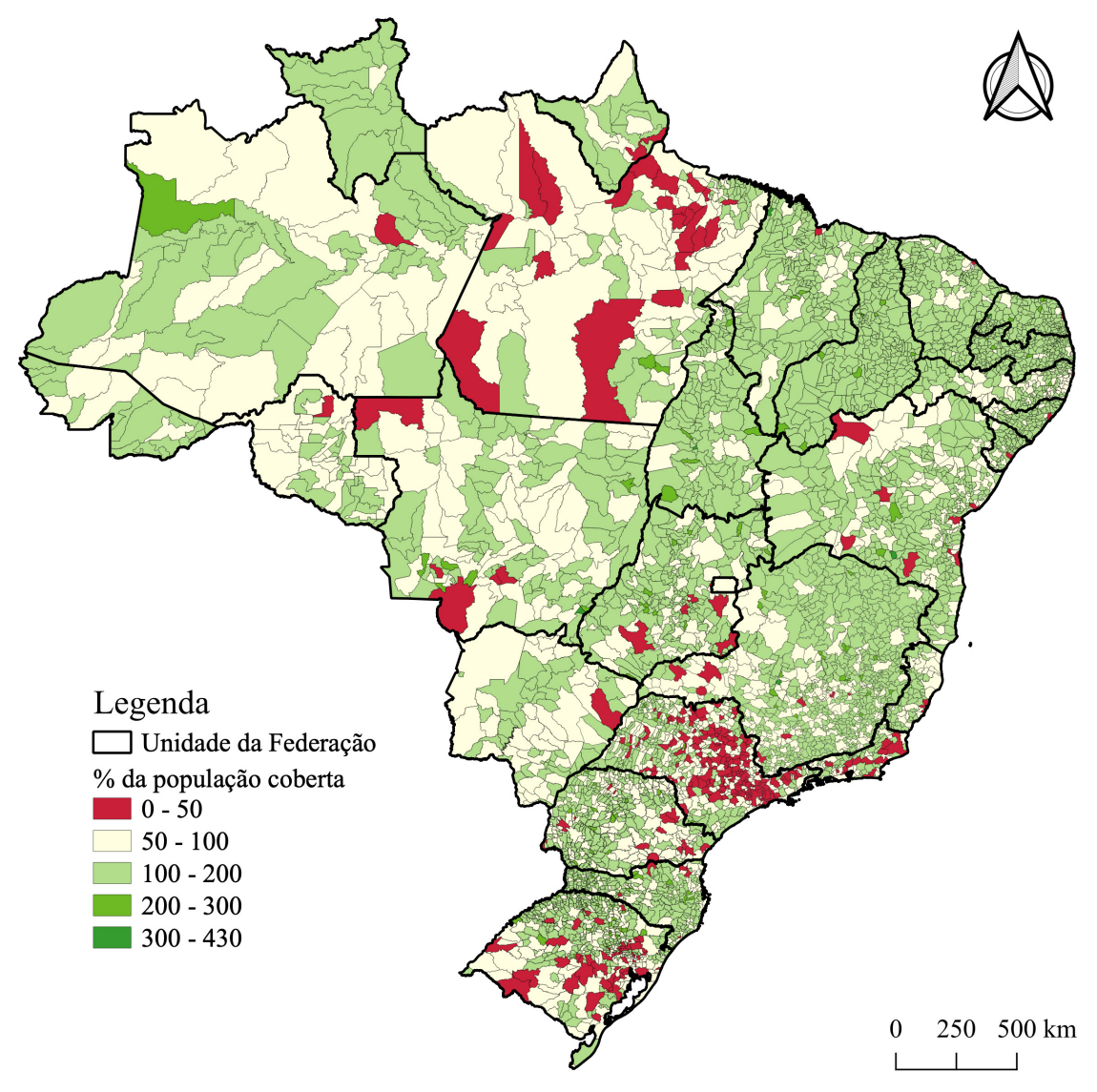

Figura 1. Cobertura potencial da população municipal por equipes de saúde, Brasil, 2018.

Nota: Foi considerado o parâmetro de 3.450 pessoas cobertas por equipe de saúde.

Fonte: Elaborado pelos autores com base nos dados do Sistema de Informação da Atenção Básica (SIAB) e do IBGE com malha digital municipal do IBGE.

Os dados constantes na Figura 1 sugerem ainda um padrão de concentração de municípios com alta cobertura em alguns estados do Nordeste e de baixa con- 
centração no Pará, em São Paulo e no Rio Grande do Sul. Ao analisar a população descoberta, a Figura 2, a seguir, apresenta os percentuais de população que excedem a capacidade de cobertura das equipes de saúde nos municípios brasileiros. À exceção de Cachoeira do Arari e Jacareacanga, no Pará, todos aqueles com mais de $80 \%$ da população descoberta se encontram nas regiões Sul e Sudeste, resultado que aponta para a necessidade de avaliar a cobertura em paralelo com o nível de renda média. Isso porque, conforme demonstrado por Carvalho (2017), essas regiões apresentam os menores percentuais de população dependente do SUS no país. Portanto, a baixa cobertura em algumas regiões pode indicar a priorização na alocação de investimento público decorrente da concorrência com o serviço privado, em um efeito substituição. A fim de contornar essa possibilidade, a análise intramunicipal apresentada na próxima seção leva em conta também a renda domiciliar média como indicativo da dependência do SUS.

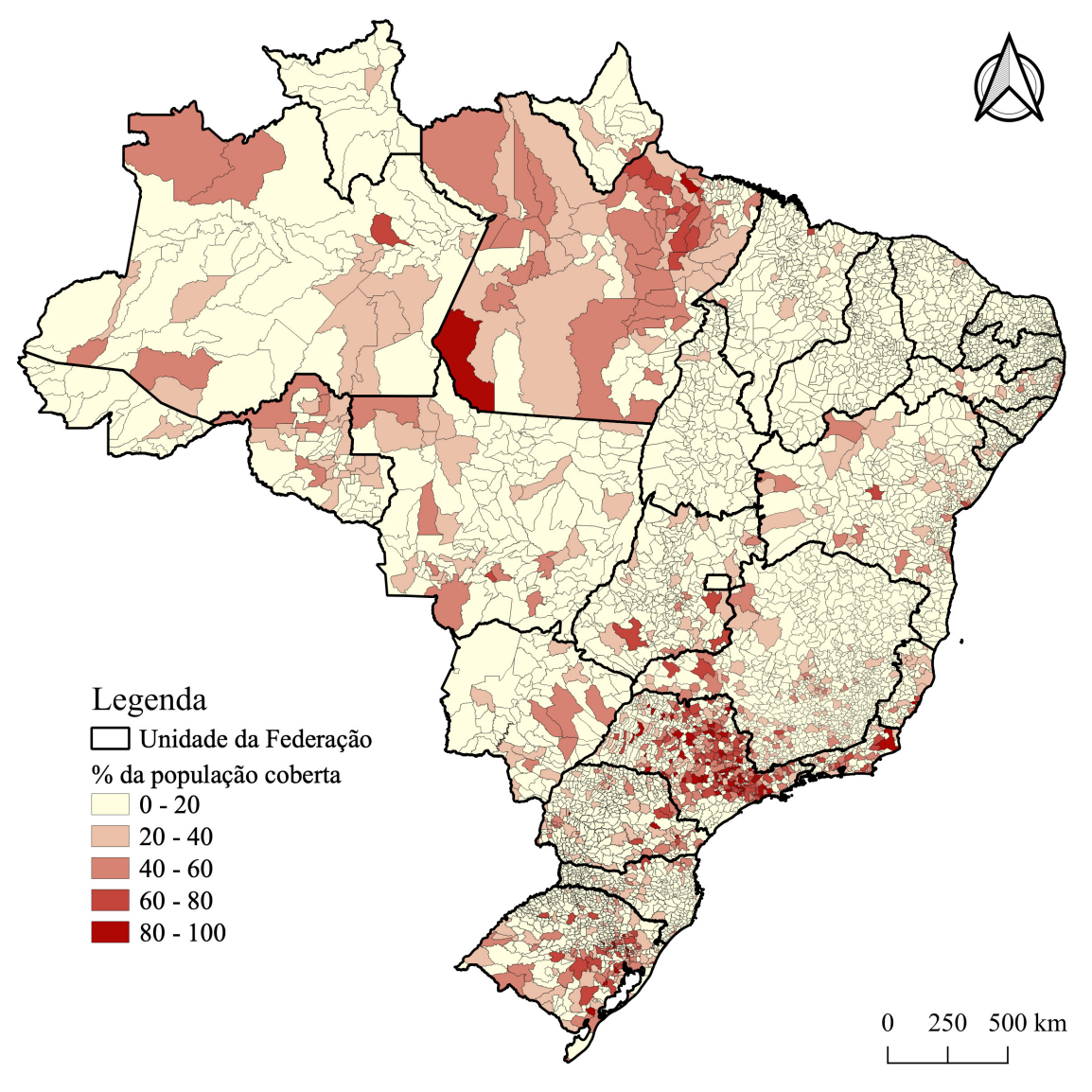

Figura 2. Percentual da população descoberta por equipes de saúde, Brasil, 2018. Nota: Foi considerado o parâmetro de 3.450 pessoas cobertas por equipe de saúde. Fonte: Elaborado pelos autores com base nos dados do SIAB e do IBGE com malha digital municipal do IBGE. 
A distribuição espacial do percentual da população descoberta (estatisticamente pseudossignificativa a 5\%) pode ser observada na Figura 3. Nela, destaca-se a formação dos agrupamentos do tipo Baixo-Baixo nos estados do Pará, São Paulo e Rio Grande do Sul, além do Distrito Federal, com baixa oferta de equipes nesses clusters municipais.

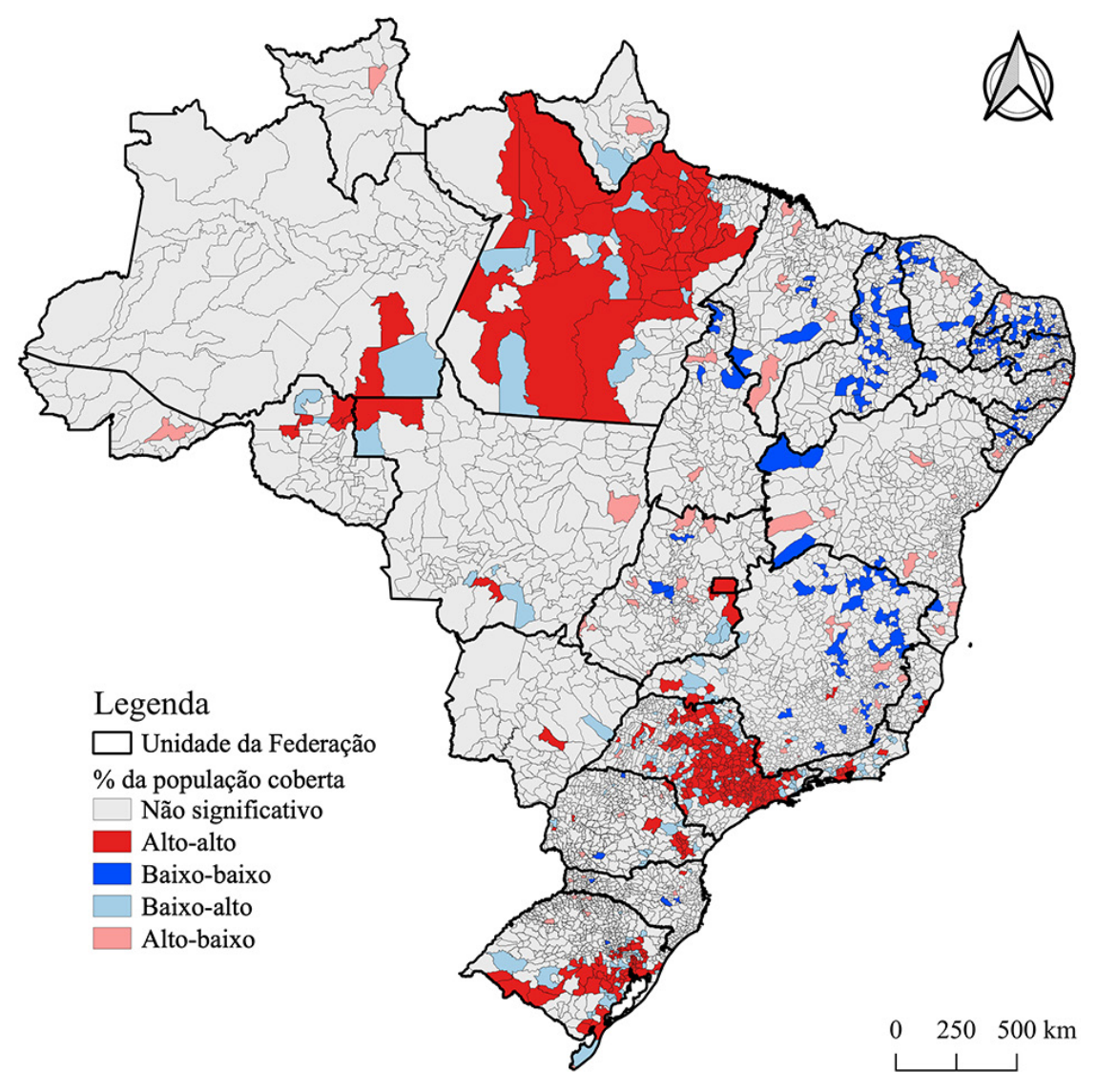

Figura 3. Associação espacial do percentual da população descoberta por equipes de saúde, Brasil, 2018.

Nota: Foi considerado o indicador I de Moran Local calculado a partir de matriz de vizinhança do tipo Rainha de primeira ordem.

Fonte: Elaborado pelos autores com base nos dados do SIAB e do IBGE com malha digital municipal do IBGE.

O resultado encontrado contrasta com estudos de oferta de equipamentos de média e alta complexidade. Conforme apresentado por Rocha et al. (2017) e Amaral et al. (2017), a provisão de serviços de média e alta complexidade apresenta baixa cobertura nas regiões Norte, Centro-Oeste e Nordeste. Já em termos de atenção primária, constata-se que a baixa cobertura se concentra principalmente nas regiões Norte, Sudeste e Sul. Enquanto o percentual de população 
dependente do SUS chega a 90\% na região Norte, têm-se números inferiores a $60 \%$ no estado de São Paulo e a 70\% no Rio Grande do Sul (CARVALHO, 2017). Parece-nos, portanto, que há dois processos distintos evidenciados pela baixa cobertura de atenção primária nas regiões brasileiras. O primeiro é o vazio assistencial absoluto em regiões onde a provisão pelo sistema público é primaz, como é o caso da região Norte. O segundo, por sua vez, é um vazio relativo em regiões onde pode haver um efeito substituição a partir da provisão privada, como se verifica nas regiões Sul e Sudeste.

É importante frisar que, mesmo em municípios em que a quantidade de equipes de saúde é suficiente, é possível que elas estejam excessivamente concentradas no que se refere ao espaço, favorecendo algumas áreas em detrimento de outras. E mais, nas cidades em que a cobertura é insuficiente para atender a população, é primordial identificar exatamente as regiões desatendidas, mediante uma análise intramunicipal, foco da próxima seção. Assim como para a análise intermunicipal, é relevante na análise intramunicipal identificar as regiões, no caso os setores censitários, carentes de cobertura em paralelo com uma análise de renda média para qualificar as populações descobertas, uma vez que elas podem ser descobertas em absoluto ou apenas de provisão pública, com cobertura por provisão de serviços privados.

\subsection{Análise intramunicipal - estudo em Belo Horizonte, Minas Gerais}

A análise intramunicipal aplicada no município de Belo Horizonte, capital de Minas Gerais, permitiu identificar, segundo os dados geolocalizados e a população dos setores censitários, um total de quase 487 mil pessoas sem cobertura, ou 20,6\%. Em 2000, Belo Horizonte contava com 545 equipes geolocalizadas e 2,37 milhões de habitantes (em 2018, segundo dados do DAB, seriam 578 equipes e população estimada pelo IBGE de 2,51 milhões).

A Figura 4 mostra a localização dos setores censitários onde se concentra a população descoberta e também indica os setores que cada UBS do município cobre. Além disso, pode-se perceber grande concentração de setores descobertos na região central da cidade, reconhecidamente de alta renda, e na região sul, o que evidencia a necessidade de qualificar a situação de cobertura com base no nível de renda na região. Buscando essa qualificação, e tendo em vista que habitantes dos setores censitários de alta renda potencialmente contam com acesso a saúde suplementar, enquanto aqueles que habitam setores censitários de baixa renda tendem a ser mais dependentes exclusivamente do SUS, ressaltam-se aqui os setores de baixa em renda em relação aos demais. Assim, na Figura 4, os setores com renda média inferior a $\mathrm{R} \$$ 510,00 são apresentados em amarelo e se localizam, em 
sua maioria, nos extremos do município, áreas, em geral, com cobertura satisfatória de equipes.

Assim, é possível, com o apoio do resultado apresentado, identificar com precisão as áreas carentes de cobertura, bem como aquelas em que a cobertura é aparentemente suficiente, em concomitância com as características de renda dos setores censitários.

Há que ressaltar que a demanda é mensurada neste trabalho como tamanho da população. Conforme apresentado por Azevedo (2014), a demanda por atenção primária ainda é afetada pela própria forma de ocupação da cidade e por suas centralidades de atratividade populacional com fins não residenciais, como centros de comércio ou concentração de trabalho. Nesses casos, a circulação de pessoas pode elevar a demanda por atenção primária à saúde.

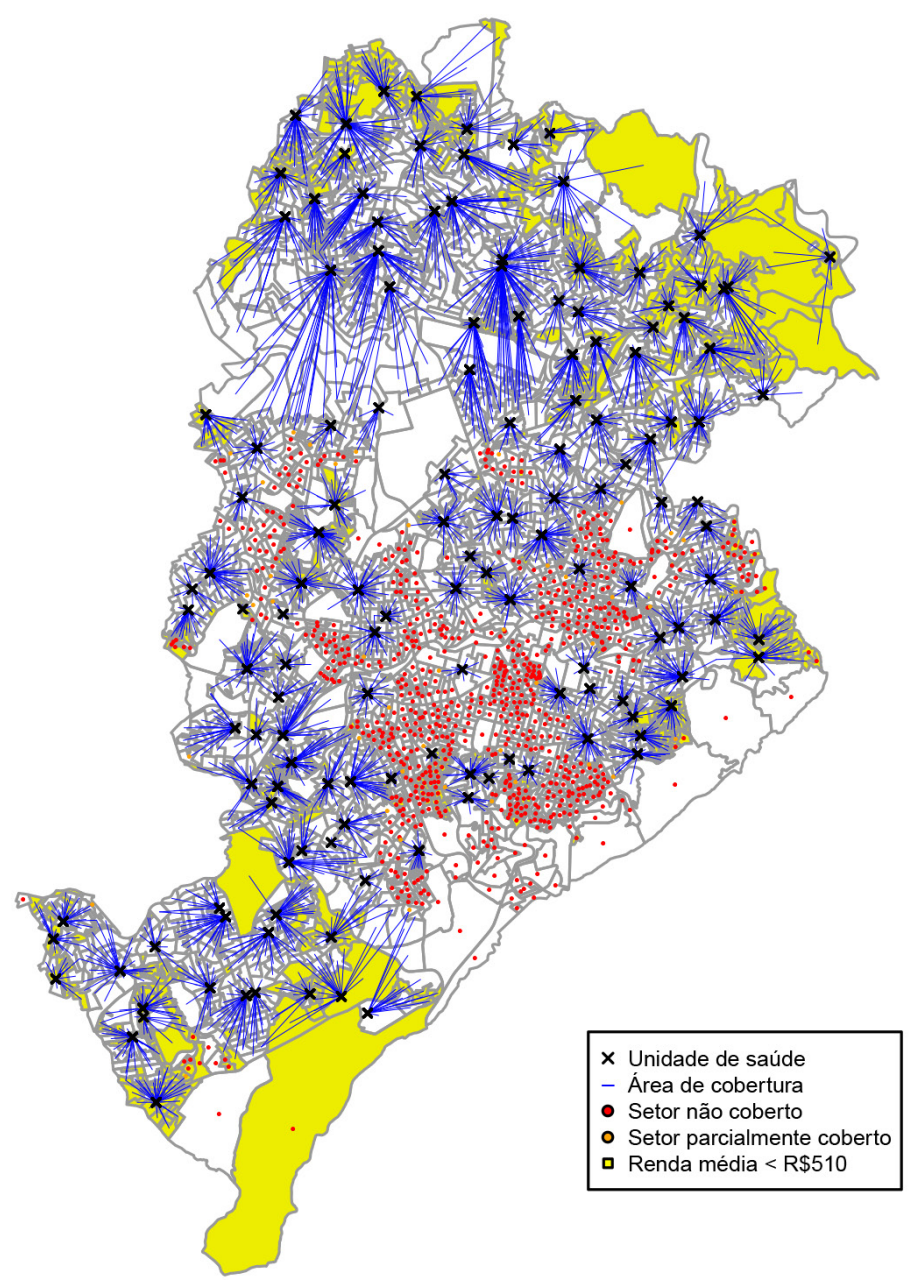

Figura 4. Cobertura potencial intramunicipal e renda média, por setores censitários, Belo Horizonte, 2018

Fonte: Elaborado pelos autores com base nos dados do SIAB e do IBGE com malha digital dos setores censitários do IBGE. 
O resultado apresentado para o município de Belo Horizonte é um exemplo de que as equipes de saúde podem não estar distribuídas de maneira equitativa pelo território. Na análise feita, por exemplo, o setor censitário em amarelo localizado no extremo sul da cidade, ocupado por pessoas de baixa renda, não é potencialmente atendido por nenhuma equipe do Programa Saúde da Família. Ou seja, em função da capacidade de atendimento das equipes e da população em setores mais próximos à UBS, a capacidade de cobertura da UBS mais próxima seria potencialmente insuficiente para atender aos moradores do setor censitário em questão. Desde modo, os resultados encontrados podem sugerir, em contrapartida, que o município com número insuficiente de equipes poderia direcionar sua oferta para setores censitários com maiores fragilidades socioeconômicas, em detrimento daqueles com maior nível de renda.

Um qualificador importante é a distância entre a unidade e o local de residência da população, o que pode dificultar o acesso, mesmo em áreas que se encontram sob a cobertura de uma unidade de saúde. É o caso, principalmente, da região norte de Belo Horizonte, como se vê na Figura 4. Souza et al. (2016) apresentam as áreas de abrangência dos centros de saúde de Belo Horizonte utilizando limites de quinze ou trinta minutos de caminhada. Diante da heterogeneidade topográfica da cidade e das possibilidades de acesso, a distância entre o ponto de provisão e o de residência da população pode dificultar a acessibilidade a esses centros.

Especificamente em relação à pandemia da Covid-19, cabe ressaltar, mais uma vez, que o protocolo estabelecido pelo SUS prevê atenção à saúde pela rede primária nos estágios iniciais da doença, independentemente do nível de gravidade (BRASIL, 2020). Dessa forma, considerou-se neste trabalho o total da população sem qualificação por estrutura etária ou sexo. Todavia, para os demais níveis de complexidade de atenção, como atenção hospitalar, faz-se necessário categorizar essas variáveis como qualificadoras da demanda e da acessibilidade, uma vez que as taxas de internação e de duração da internação variam significativamente de acordo com a idade do paciente (PEREIRA et al., 2020a).

Em relação às áreas não cobertas, aqui identificadas para fins de priorização de investimentos, é necessária uma avaliação no que diz respeito à melhor forma de ampliação da cobertura, seja por meio de novas unidades de saúde, seja mediante a ampliação das existentes ou ainda a readequação da provisão. No cenário da pandemia de Covid-19, Tasca e Massuda (2020) demonstram isso, por exemplo, ao analisar as estratégias adotadas pelos gestores de saúde da rede pública da região do Lácio, na Itália, onde foi preciso modificar o modo de funcionamento tanto pela abordagem dos profissionais de saúde como pelo uso de formas remotas de atenção. Já Vitória e Campos (2020) apontam que os casos se distribuem no 
território brasileiro e que se espera que $80 \%$ deles sejam atendidos por telefone ou o mais próximo possível dos domicílios. Ou seja, há diferentes caminhos para ampliar a cobertura dos serviços de APS, mesmo em um contexto de crise, como é o caso da pandemia de Covid-19.

\section{Considerações finais}

Este trabalho destaca a necessidade de qualificar a distribuição das equipes de saúde da família em escala intramunicipal como ponto de partida para uma organização da atenção primária eficientemente distribuída no território.

Estudos dessa natureza, além do potencial para subsidiar políticas públicas ou o aperfeiçoamento da gestão, sugerem reflexões sobre a lógica do sistema de saúde. Com efeito, a análise sobre eficiência e ineficiência não deve marginalizar compreensões da realidade, nem construir realidades falsas e deixar de lado aspectos de relevância para a avaliação da organização de saúde (CUNHA, 2011).

Também se deve ponderar sobre o contexto atual da pandemia da Covid-19 e seus efeitos sobre a APS, em face de seu papel como principal meio de acesso à saúde. Tem sido observado que os serviços de saúde especializados não seriam suficientes para enfrentar com sucesso a propagação da pandemia (REDE APS; ABRASCO 2020), o que reforça o papel da Saúde da Família com sua abordagem comunitária e territorial.

Isso reforça as premissas abordadas pelo estudo, quer em situações extremas, como a da pandemia, quer naquelas observadas em ambiente de relativa normalidade. Nesta perspectiva, a definição de políticas públicas na saúde, utilizando como norteadores estudos voltados à eficiência alocativa, pode proporcionar resultados mais equitativos do que aquelas políticas com ênfase em escala e escopo, que tomam como fator de decisão a fronteira de eficiência. Essa discussão, embora neste artigo se restrinja à organização da atenção primária, mostra-se fundamental no tocante aos hospitais, de pequeno porte ou não, à oferta de recursos humanos e aos diferentes níveis de atenção (média e alta complexidade). Dada a integralidade do SUS, tem-se que o fortalecimento do sistema, em todos os seus níveis, apresenta-se como crucial para o aprimoramento da capacidade de monitoramento, acompanhamento e acolhida em situação de choques de demanda, como o enfrentado durante a pandemia de Covid-19.

Por fim, a disponibilidade de dados em âmbito local, associados aos indicadores de saúde e sociais, podem melhorar a análise em direção a estimativas mais próximas da realidade, permitindo uma alocação mais adequada para reduzir iniquidades na oferta e no acesso aos serviços de saúde. 


\section{Referências}

ADAY, L.; ANDERSEN, R. A framework for the study of access to medical care. Health services research, v. 9, n. 3, p. 208-220, 1974.

AMARAL, P. Spatial structure of health equipment in Brazil. 2013. Tese (Doutorado) - University of Cambridge, Cambridge, 2013.

AMARAL, P. et al. Spatially balanced provision of health equipment: a cross-sectional study oriented to the identification of challenges to access promotion. International Journal for Equity in Health, v. 16, p. 1-13, 2017.

ANDRADE, M. V. et al. Brazil's Family Health Strategy: factors associated with programme uptake and coverage expansion over 15 years (1998-2012). Health Policy and Planning, v. 33, n. 3, p. 368-380, 2018a.

. et al. Transition to universal primary health care coverage in Brazil: analysis of uptake and expansion patterns of Brazil's Family Health Strategy (1998-2012). PloS one, v. 13, n. 8, p. e0201723, 2018b.

ANSELIN, L. Local Indicator of Spatial Association - LISA. Geographical Analysis, v. 27, n. 3, p. 93-115, 1995.

AZEVEDO, S. J. S. Segregação e oportunidades de acesso aos serviços básicos de saúde em Campinas: vulnerabilidades sociodemográficas no espaço intraurbano. Campinas: Núcleo de Estudos de População; Unicamp, 2014.

BARBOSA, A. et al. Programa Mais Médicos: como avaliar o impacto de uma abordagem inovadora para superação de iniquidades em recursos humanos. Revista Panamericana de Salud Publica-Pan American Journal of Public Health, v. 42, p. 1, 2018.

BRASIL. MINISTÉRIO DA SAÚDE. SAPS. Protocolo de manejo clínico do coronavírus (Covid-19) na Atenção Primária à Saúde. Ministério da Saúde; Secretaria de Atenção Primária à Saúde, 2020. [Internet] Disponível em: https://portaldeboaspraticas.iff.fiocruz.br/biblioteca/protocolo-de-manejo-clinico-do-coronavirus-covid-19-na-atencao-primaria-a-saude/. Acesso em: 27 jan. 2021.

CARVALHO, L. Distribuição espacial da oferta de saúde no Brasil no contexto do mix público-privado. Dissertação (Mestrado em Economia) - Faculdade de Ciências Econômicas, Universidade Federal de Minas Gerais, Belo Horizonte, 2017. p. 96. Disponível em: http:// hdl.handle.net/1843/FACE-AMFJX8. Acesso em: 27 jan. 2021.

CARVALHO, V. K. da S.; MARQUES, C. P.; SILVA, E. N. da. A contribuição do Programa Mais Médicos: análise a partir das recomendações da OMS para provimento de médicos. Ciência \& Saúde Coletiva, v. 21, n. 9, p. 2773-2784, 2016.

CHRISTÄLLER, W. Central places in Southern Germany. Englewood Cliff: Prentice-Hall, 1966.

CUNHA, J. A. C. Avaliação de desempenho e eficiência em organizações de saúde: um estudo em hospitais filantrópicos. 2011. Tese (Doutorado) - Faculdade de Economia, Administração, Contabilidade e Atuária, Universidade de São Paulo, São Paulo, 2011.

DRANOVE, D.; WHITE, W. D.; WU, L. Segmentation in local hospital markets. Medical Care, v. 31, n. 1, p. 52-64, 1993 
GOODMAN, D. C. et al. The distance to community medical care and the likelihood of hospitalization: is closer always better? American Journal of Public Health, v. 87, n. 7, p. 1144-1150, jul. 1997.

GUIMARÃES, R. B. Saúde urbana: velho tema, novas questões. Terra Livre, v. 2, n. 17, p. 155-170, 2001.

HAMER, L. Improving patient access to health services: a national review and case studies of current approaches. London: Health Development Agency, 2004.

HART, J. The inverse care law. Lancet, i, p. 405-412, 1971.

HIGGS, G. The role of GIS for health utilization studies: literature review. Health Services and Outcomes Research Methodology, v. 9, n. 2, p. 84-99, 2009.

IUNES, R. A concepção econômica de custos. In: PIOLA, S.; VIANNA, S. (ed.). Economia da saúde: conceito e contribuição para a gestão da saúde. 3. ed. Brasília, DF: Ipea, 2002. p. 227-247.

JOSEPH, A.; BANTOCK, P. Measuring potential physical accessibility to general practitioners in rural areas: a method and case study. Social Science and Medicine, v. 16, n. 1, p. 85-90, 1982.

KHAN, A. An integrated approach to measuring potential spatial access to health care services. Socio Economic Planning Sciences, v. 26, n. 4, p. 275-287, 2002.

KUHN, M.; OCHSEN, C. Demographic and geographic determinants of regional physician supply, 2009, Barcelona. III WORLD CONFERENCE OF THE SPATIAL ECONOMETRICS ASSOCIATION. Proceedings [...]. Barcelona: Spatial Econometrics Association, 2009.

LÖSCH, A. The economics of location. London: Yale University, 1954.

NORONHA, K. Dois ensaios sobre desigualdade social em saúde. 2001. Dissertação (Mestrado) - Universidade Federal de Minas Gerais, Belo Horizonte, 2001.

OLIVEIRA, E. X. G. de; TRAVASSOS, C.; CARVALHO, M. S. Acesso à internação hospitalar nos municípios brasileiros em 2000: territórios do sistema único de saúde. Cadernos de Saúde Pública, Rio de Janeiro, v. 20, sup., n. 2, p. S298-S309, 2004.

PEREIRA, R. et al. Geographic access to Covid-19 healthcare in Brazil using a balanced float catchment area approach. medRxiv, 2020a. DOI: 2020.07.17.20156505. Disponível em: https://www.medrxiv.org/content/10.1101/2020.07.17.20156505v1. Acesso em: 27 jan. 2021.

Desigualdades socioespaciais de acesso a oportunidades nas cidades brasileiras 2019. Brasília, DF: Ipea, 2020b (Texto para discussão, n. 2535).

POL, L.; THOMAS, R. The demography of health and health care. 2nd ed. New York: Plenum, 2000.

PÓVOA, L.; ANDRADE, M.; MORO, S. Distribuição dos empregos médicos em Minas Gerais: uma análise à luz da economia espacial, 2004, Diamantina. XI SEMINÁRIO SOBRE A ECONOMIA MINEIRA. Anais [...]. Diamantina: Cedeplar/UFMG, 2004.

RAHMAN, S.; SMITH, D. Use of location-allocation models in health service development planning in developing nations. European Journal of Operational Research, v. 123, p. 437-452, 2000. 
REDE APS. REDE DE PESQUISA EM ATENÇÃO PRIMÁRIA À SAÚDE; ABRASCO. ASSOCIAÇÃO BRASILEIRA DE SAÚDE COLETIVA. Fortalecer la Atención Primaria en Salud y la estrategia Salud de la Familia para el enfrentamiento de la Covid-1g en el SUS: posicionamiento de la Red de investigación en Atención Primaria en Salud (Rede APS). [Internet] Rio de Janeiro, 2020. Disponível em: https://redeaps.org.br/wp-content/uploads/2020/o6/APSSUS-Brasil-Covid-19-Rede-APS-ABRASCO-esp.pdf. Acesso em: 27 jan. 2021.

RIBEIRO, H.; SILVA, E. N. Desigualdades intraurbanas em internações hospitalares por doenças respiratórias e circulatórias em uma área da cidade de São Paulo. Cadernos Metrópole, v. 18, n. 36, p. 461-480, 2016.

ROCHA, T. et al. Access to emergency care services: a transversal ecological study about Brazilian emergency health care network. Public Health, v. 153, p. 9-15, 2017.

Geolocalização de internações cadastradas no Sistema de Informações Hospitalares do Sistema Único de Saúde: uma solução baseada no programa estatístico R. Epidemiologia e Serviços de Saúde. v. 27, n. 4, 2018.

SAPS. SECRETARIA DE ATENÇÃO PRIMÁRIA À SAÚDE. Relatório de gestão. Brasília, DF: Ministério da Saúde, 2020.

SERVO, L.; ANDRADE, M.; AMARAL, P. do. Análise das regiões de saúde no Brasil a partir do Pacto pela Saúde: adequação da regionalização e acesso geográfico. XXI ENCONTRO NACIONAL DE ESTUDOS POPULACIONAIS, 2018, Poços de Caldas. Anais [...]. Poços de Caldas: Abep, set. 2018. Tema: População, Sociedade e Políticas: desafios presentes e futuros.

SOUZA, R. C. F. et al. Viver próximo à saúde em Belo Horizonte. Cadernos Metrópole, São Paulo, v. 18, n. 36, p. 325-344, 2016.

STOCK, R. Distance and the utilization of health facilities in rural Nigeria. Soc. Sci. Med., v. 17, n. 9, p. 563-570, 1983.

TASCA, R.; MASSUDA, A. Estratégias para reorganização da rede de Atenção à Saúde em resposta à pandemia Covid-19: a experiência do sistema de saúde italiano na região de Lazio. APS em Revista, especial Covid, v. 2, n. 1, p. 20, jan.-abr. 2020. DOI: 10.14295/aps. v2i1.65

VITÓRIA, Â. M.; CAMPOS, G. W. de S. Só com APS forte o sistema pode ser capaz de achatar a curva de crescimento da pandemia e garantir suficiência de leitos UTI. Frente Estamira de CAPS [Internet] 2 abr. 2020. Rio de Janeiro, 2020. Disponível em: https://frenteestamira.org/2020/04/o2/so-com-aps-forte-o-sistema-pode-ser-capaz-de-achatar-a-curva-de-crescimento-da-pandemia-e-garantir-suficiencia-de-leitos-uti/. Acesso em: 27 jan. 2021.

WHITEHEAD, M. The concepts and principles of equity and health. International Journal of Health Services: Planning, Administration, Evaluation, v. 22, n. 3, p. 429-445, 1992.

WHO. Health in 2015: from MDGs, Millennium Development Goals to SDGs, Sustainable Development Goals. Geneva: World Health Organization, 2015. 


\section{Pedro Amaral}

Graduado em Ciências Econômicas pela Universidade Federal de Minas Gerais (UFMG), Mestre em Economia pelo Cedeplar/UFMG e Ph.D. pela University of Cambridge. Professor do Departamento de Ciências Econômicas da UFMG (Cedeplar/UFMG) e fellow do Center for Spatial Data Science (University of Chicago). Foi secretário adjunto (20152017), secretário executivo (2017) e membro suplente do Conselho Fiscal (2017-2019) da Associação Nacional de Pós-graduação e Pesquisa em Planejamento Urbano e Regional (ANPUR).

Email: pedroamaral@cedeplar.ufmg.br

ORCID: 0000-0002-2505-035X

Contribuição de autoria: conceituação; análise formal; investigação/pesquisa; metodologia; visualização; escrita - primeira redação; escrita - revisão e edição.

\section{Lucas Resende de Carvalho}

Graduado em Ciências Econômicas pela Universidade Federal de Minas Gerais (UFMG), com mestrado em Economia pelo Centro de Desenvolvimento e Planejamento Regional da Faculdade de Ciências Econômicas da UFMG. Doutorando do Programa de Pós-graduação em Economia da mesma instituição.

Email: lucasrc@cedeplar.ufmg.br

ORCID: 0000-0002-3618-3967

Contribuição de autoria: curadoria de dados; análise formal; investigação/pesquisa; metodologia; escrita - primeira redação; escrita - revisão e edição. 


\section{Luciana Luz}

Graduada em Ciências Econômicas pela Universidade Federal de Minas Gerais (UFMG), com mestrado em Demografia pelo Centro de Desenvolvimento e Planejamento Regional da Faculdade de Ciências Econômicas da mesma instituição. Ph.D. em Sociologia/ Demografia pela Arizona State University. Professora adjunta do Departamento de Demografia da UFMG e pesquisadora do Cedeplar.

Email: lucianaluz@cedeplar.ufmg.br

ORCID: 0000-0002-0623-1438

Contribuição de autoria: conceituação; análise formal; investigação/pesquisa; escrita - primeira redação; escrita - revisão e edição.

\section{Allan Claudius Queiroz Barbosa}

Graduado em Ciências Econômicas pela Pontifícia Universidade Católica de Minas Gerais (PUC Minas), mestre em Administração pela Universidade Federal de Minas Gerais (UFMG) e doutor em Administração pela Universidade de São Paulo (USP). Professor titular da UFMG e professor residente do Instituto de Estudos Avançados Transdisciplinares (IEAT) da mesma instituição.

Email: allan@ufmg.br

ORCID: 0000-0003-1266-5168

Contribuição de autoria: conceituação; análise formal; investigação/pesquisa; escrita - primeira redação; escrita - revisão e edição.

Submissão: 23 de julho de 2020.

Aprovação: 3 de fevereiro de 2021.

Como citar: AMARAL, P.; CARVALHO, L. R.; LUZ, L. S.; BARBOSA, A. C. Q. Estrutura espacial e provisão de atenção primária à saúde nos municípios brasileiros. Revista brasileira de estudos urbanos e regionais. v. 23, E202110, 2021. DOI 10.22296/2317-1529.rbeur.202110

Artigo licenciado sob Licença Creative Commons CC BY 4.0. https://creativecommons.org/licenses/by/4.o/deed.pt_BR 
ERRATA

No artigo Estrutura espacial e provisão de atenção primária à saúde nos municípios brasileiros, com número DOI 10.22296/2317-1529.rbeur.202110, publicado na Revista Brasileira de Estudos Urbanos e Regionais, v. 23, E202110, 2021:

Ná página 4

No título, onde se lê:

Estrutura espacial e provisão de atenção primária à saúde nos municípios brasileiros

Leia-se:

Estrutura espacial e provisão de atenção primária à saúde nos municípios brasileiros $^{1}$

Nota de rodapé

1. Os autores agradecem à Fapemig pelo apoio recebido por meio do projeto APQ-04005-17

Ná página 10

Na nota de rodapé, onde se lê:

1.

Leia-se:

2. 